\title{
Noonan Syndrome and Acute Myeloid Leukemia in Adults: The Importance of a Correct Multidisciplinary Approach during Childhood
}

\author{
Fabio Timeus \\ Department of Pediatrics, Chivasso Hospital, Turin, Italy
}

\section{Editorial comment on the paper by Alhumaid et al. Comprehensive Genomic Analysis of Noonan Syndrome and Acute Myeloid Leukemia in Adults: A Review and Future Directions. A Case Report. Acta Haematol 2020;143:583-593}

In this issue of Acta Haematologica, Alhumaid et al. [1] describe the rare case of an adult with Noonan syndrome (NS) who developed acute myeloid leukemia (AML) characterized by a combination of a germline mutation of PTPN11 with somatic mutations of NPM1, IDH1, and $B C L 6$. NS is an autosomal dominant disorder functionally characterized by hyperactivation of the RAS/MAPK signaling pathway, caused by mutations in various genes, the most frequent being PTPN11, mutated in about $50 \%$ of cases. The PTP11 gene encodes for the nonreceptor protein tyrosine phosphatase SHP-2, and germline and somatic mutations of PTPN11 confer a gain-of-function of SHP-2. The NS phenotype is characterized by short stature, multiple dysmorphisms, congenital heart defects, cryptorchidism, coagulopathy, lymphatic dysplasia, and mild mental impairment. NS patients are at an increased risk of developing cancers (from 3.5 to 8 times in different series), the most frequent of which is juvenile myelomonocytic leukemia (JMML). Other cancers observed in NS are acute myelogenous leukemia (AML), acute lymphoblastic leukemia, rhabdomyosarcoma, and neuroblastoma. A myeloproliferative disorder similar to JMML but with a spontaneous remission has been described in NS [2].

The case report by Alhumaid et al. [1] suggests several considerations. First is the importance of an early diag-

\footnotetext{
karger@karger.com

(c) 2020 S. Karger AG, Basel

www.karger.com/aha

Karger!
}

nosis and a correct multidisciplinary approach during childhood. Among congenital syndromes, NS has the highest frequency of cardiac defects after trisomy 21, and the surgical correction of the atrial septal defect would have modified the clinical course of the reported patient. A correct transition from pediatric to adult care is critical in patients with chronic diseases.

A second point is the recommendation for cancer surveillance of RASopathies in patients with high-risk mutations. In this regard, Tartaglia et al. [3] analyzed germline and somatic PTPN11 mutations and demonstrated that the cancer risk is related to the level of SHP2 tyrosin phosphatase activity. In NS patients with highrisk mutations, clinical and laboratory surveillance every 3-6 months for the first 5 years is recommended [4]. A noninvasive functional evaluation of peripheral blood hematopoietic progenitors has also been suggested for high-risk subjects [5]. Cancer prevention strategies also play a role in subjects at risk of developing malignancies, with the focus on lifestyle and environmental factors. The PTPN11 mutation p.Phe285Ser observed in the case report has been described in JMML, so the patient could be considered to be at risk of malignant transformation.

In adult AML, somatic mutations of PTPN11 are often associated with NPM1 mutations, according to the two- 
hit theory of leukemogenesis, with those patients mutated in PTPN11 but not in NPM1 showing a poorer prognosis [6]. The presence of other co-occuring mutations may further influence the clinical course and prognosis [7]. The genomic analysis performed in the patient described by Alhumaid et al. [1] showed the presence of a combination of a germline mutation of PTPN11 with somatic mutations of NPM1, IDH1, and BCL6. The disease relapse in the presence of this complex genomic profile could suggest an intensive treatment including allogeneic stem cell transplantation (HSCT). Successful HSCT has been reported in an adult NS patient with AML in second remission [8]. Unfortunately, the clinical conditions and comorbidities of the patient described by Alhumaid et al. [1] made this approach impossible.
Analysis of single-nucleotide polymorphisms and whole-genome sequencing now make it possible to revisit the classification of AML on a purely genomic basis, and to shift in the near future to precision medicine with targeted therapies of particular interest in patients with severe comorbidities.

\section{Disclosure Statement}

The author has no conflicts of interest to declare.

\section{Funding Sources}

There was no funding for the writing of this manuscript.

\section{References}

1 Alhumaid M, Dasouki MJ, Ahmed SO, Abalkhail H, Hagos S, Wakil S, et al. Comprehensive genomic analysis of Noonan syndrome and acute myeloid leukemia in adults: a review and future directions. Acta Haematol. 2020. DOI: 10.1159/000505715.

2 Roberts AE, Allanson JE, Tartaglia M, Gelb BD. Noonan syndrome. Lancet. 2013 Jan; 381(9863):333-42.

3 Tartaglia M, Martinelli S, Stella L, Bocchinfuso G, Flex E, Cordeddu V, et al. Diversity and functional consequences of germline and somatic PTPN11 mutations in human disease. Am J Hum Genet. 2006 Feb;78(2):27990.
4 Villani A, Greer MC, Kalish JM, Nakagawara A, Nathanson KL, Pajtler KW, et al. Recommendations for Cancer Surveillance in Individuals with RASopathies and Other Rare Genetic Conditions with Increased Cancer Risk. Clin Cancer Res. 2017 Jun;23(12):e83-90.

5 Timeus F, Crescenzio N, Baldassarre G, Doria A, Vallero S, Foglia L, et al. Functional evaluation of circulating hematopoietic progenitors in Noonan syndrome. Oncol Rep. 2013 Aug;30(2):553-9.
6 Hou HA, Chou WC, Lin LI, Chen CY, Tang JL, Tseng MH, et al. Characterization of acute myeloid leukemia with PTPN11 mutation: the mutation is closely associated with NPM1 mutation but inversely related to FLT3/ITD. Leukemia. 2008 May;22(5):1075-8.

7 Papaemmanuil E, Gerstung M, Bullinger L Gaidzik VI, Paschka P, Roberts ND, et al. Genomic Classification and Prognosis in Acute Myeloid Leukemia. N Engl J Med. 2016 Jun; 374(23):2209-21.

8 Matsubara K, Yabe H, Ogata T, Yoshida R, Fukaya T. Acute myeloid leukemia in an adult Noonan syndrome patient with PTPN11 mutation. Am J Hematol. 2005 Jun;79(2):171-2. 